\title{
9 TRABAJAR LAS EMOCIONES MEDIANTE B-LEARNING EN LOS FUTUROS PROFESIONALES DE ENFERMERÍA: UNA EXPERIENCIA DIFERENTE DE PROMOCIÓN EN SALUD MENTAL
}

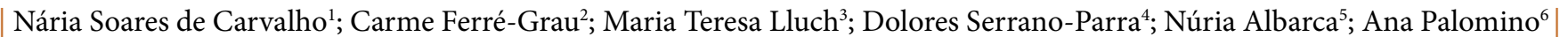

\section{RESUMEN}

CONTEXTO: Las experiencias en los espacios virtuales acostumbran a estar dirigidas a conocimientos técnicos y de aprendizaje colaborativo - cooperativo -, no siendo utilizadas para trabajar las habilidades que sean de propiedades más subjetivas, como son las emociones. Las emociones son categorizadas dentro de las competencias transversales, y son trabajadas en gran porcentaje con estrategias de aprendizaje presencial. Las emociones son de vital importancia a la hora de respuesta al estímulo. La falta de regulación puede exponer a la persona a varios riesgos en relación a su salud mental y comprometer su bienestar. Las Emociones son un elemento que interviene en el cuidado de enfermería y que puede comprometer la calidad del cuidado.

OBJETIVOS: Con el objetivo principal de estimular la regulación emocional en los futuros profesionales de enfermería - además analizar el efecto de la metodología aplicada, y verificar la satisfacción de los estudantes -, se realizó en la asignatura de enfermería de la Universidad Rovira i Virgili un proyecto piloto en la asignatura optativa de Salud Mental Positiva.

METODOLOGIA: La metodología aplicada en este estudio fue cualitativa y el método de aprendizaje fue reflexivo. El instrumento de información fue el espacio virtual de aprendizaje Moodle/ Fórum. La muestra fue de 42 estudiantes de $3^{\circ}$ del grado de enfermería.

RESULTADOS: Los estudiantes se sintieron con más libertad de expresar sus ideas y emociones ya que el espacio de aprendizaje estaba fuera de un espacio físico. También se fomentó el autoconocimiento y las relaciones interpersonales. Lo cual incidió sobre el autoconcepto (autoestima) y las emociones positivas.

CONCLUSIÓN: El uso de espacios virtuales con la metodología adecuada es una herramienta a la hora de realizar trabajos de promoción en salud mental, como el de la regulación emocional

PALAVRAS-CHAVE: B-learning; Regulación emocional; Enfermería; Salud mental

\section{RESUMEN}

“Trabalhar as emoções mediante b-learning nos futuros profissionais de enfermagem: Uma experiência diferente de promoção da saúde mental"

CONTEXTO: As experiências nos espaços virtuais costumam estar direcionadas a conhecimentos técnicos e de aprendizagem colaborativa - cooperativa -, não sendo utilizadas para trabalhar as habilidades que sejam de propriedades mais subjetivas, como são as emoções. As emoções são categorizadas dentro das concorrências transversais, e são trabalhadas em grande percentagem com estratégias de aprendizagem presencial. As emoções são de vital importância à hora de resposta ao estímulo. A falta de regulação pode expor à pessoa a vários riscos em relação a sua saúde mental e comprometer seu bem-estar. As Emoções são um elemento que intervém no cuidado de enfermagem e que pode comprometer a qualidade do cuidado.

OBJETIVO(S): Com o objetivo principal de estimular a regulação emocional nos futuros profissionais de enfermagem -além de analisar o efeito da metodologia aplicada, e verificar a satisfação dos estudantes-, realizou-se na matéria de enfermagem da Universidade Rovira i Virgili um projeto piloto na matéria optativa de Saúde Mental Positiva.

METODOLOGIA: A metodologia aplicada neste estudo foi qualitativa e o método de aprendizagem foi reflexivo. O instrumento de informação foi o espaço virtual de aprendizagem Moodle/Fórum. A amostra foi de 42 estudantes de $3^{\circ}$, do grau de enfermagem.

RESULTADOS: Os estudantes sentiram-se com mais liberdade de expressar suas idéias e emoções já que o espaço de aprendizagem estava fora de um espaço físico. Também se fomentou o autoconhecimento e as relações interperssonais. O qual incidiu sobre o autoconceito (auto-estima) e as emoções positivas.

CONCLUSÃO: $\mathrm{O}$ uso de espaços virtuais com a metodologia adequada é uma ferramenta à hora de realizar trabalhos de promoção em saúde mental, como o da regulação emocional.

\section{DESCRIPTORES: B-learning; Regulação emocional; Enferma- gem; Saúde mental}

\begin{abstract}
"Using b-learning to working on emotions with future nursing professionals: A different approach to promoting mental health"

CONTEXT: Virtual learning experiences are usually intended to foster technical knowledge and collaborative-cooperative learning, and are not used for working on more subjective skill areas such as the emotions. Emotional skills are classified among the transversal competences are usually taught in face-to-face learning environments. Emotions are of vital importance when responding to stimuluses. An inability to regulate emotions can put individuals at risk of various mental health problems and compromise their wellbeing. Emotional skills play a role in nursing and can compromise the quality of care.

OBJECTIVE: A pilot project was carried out in the optional subject Positive Mental Health of the Nursing degree at the Universitat Rovira i Virgili. The principal aims were to stimulate the emotional regulation of future nursing professionals, to analyse the efficacy of the methodology used and to determine satisfaction levels among the students.

METHODOLOGY: The methodology applied in this study was qualitative and the learning methodology was reflective. The virtual learning platform Moodle/Forum was used to deliver the methodology. The sample consisted of 42 students from the third year of the Nursing degree.

RESULTS: The students felt greater freedom to express their ideas and emotions because learning took place in a virtual rather than physical space. The methodology also boosted self-awareness and interpersonal relations, which also had an effect on self-esteem and positive emotions.

CONCLUSION: Virtual platforms used with the appropriate methodology are useful for promoting mental health and emotional regulation

\section{KEYWORDS: B-learning; Emotional regulation; Nursing; Mental health}

Submetido em 01-10-2015

Aceite em 20-03-2016
\end{abstract}

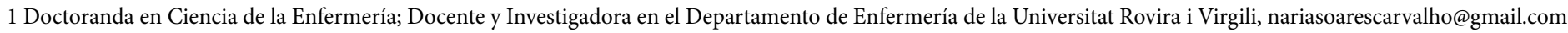

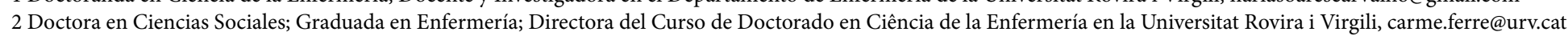
3 Doctora en Psicologia; Graduada en Enfermagem; Catedrática en Enfermería, Departamento de Enfermería de la Universitat de Barcelona, España, tlluch@ub.edu

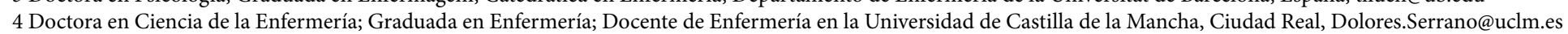
5 Doctora en Ciencia de la Enfermería; Docente de Enfermería en la Universitat Rovira i Virgili, 43002 Tarragona, España, nuria.albacar@urv.cat 6 Docente associada al Departamento de Enfermería de la Universitat Rovira i Virgili, 43002, Tarragona, España, apalomino@fundacio-puigvert.es

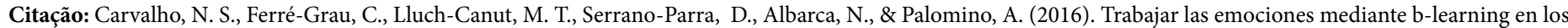
futuros profesionales de enfermería: Una experiencia diferente de promoción en salud mental. Revista Portuguesa de Enfermagem de Saúde Mental (Spe. 3), 51-56. 


\section{INTRODUCION}

La visión positiva de la salud mental tuvo punto de partida en la declaración de la Organización Mundial de la Salud (OMS), dando una nueva visión de la salud con aspectos positivos. El concepto de salud mental según la OMS se "define como un estado de bienestar en el cual el individuo es consciente de sus propias capacidades, puede afrontar las tensiones normales de la vida, puede trabajar de forma productiva y fructífera y es capaz de hacer una contribución a su comunidad".

Desde entonces, la concepción de prevención y promoción en salud física y en salud mental tuvo en cuenta aspectos más subjetivos, pero no menos importantes, vinculados al bienestar. Tales como: la felicidad, el humor, los sentimientos y emociones, el autoconcepto, las relaciones interpersonales, la capacidad de afrontamiento, las fortalezas humanas, la satisfacción personal...etc. En este ámbito se integraron varias disciplinas con la intención de comprender el bienestar humano, aportando diferentes visiones sobre el tema.

De manera que el concepto de bienestar se emplea para definir muchos estados, como por ejemplo: bienestar subjetivo. La definición está relacionada con la buena vida, aquello que hace la vida agradable, que da la felicidad en general. Es subjetiva, puesto que esta visión de bienestar es personal. Los conceptos relacionados son emociones agradables, juicios sobre uno mismo, la satisfacción con la vida o dominio en alguna faceta de la vida como trabajo, relaciones sociales...etc. (Diener, 2000).

Bienestar psicológico es el funcionamiento óptimo personal, el cual está compuesto por seis aspectos: una actitud positiva hacia ti mismo (la auto-aceptación), el sentimiento de desarrollo y las ganas de mantenerlo constante (el crecimiento personal), tener un propósito y orientación en la vida (propósito vital), ser capaz de manejar entornos complejos (dominio), tener relaciones satisfactorias e íntimas incluyendo habilidades de relación interpersonales, poder de toma sus propias decisiones de manera independiente (autonomía) y relaciones positivas (Ryff, 1989).

Las emociones, desde entonces, han sido foco de varios estudios, sin embargo aunque los sentimientos y emociones se utilizan normalmente como sinónimo, presentan definiciones diferentes. Por ello es importante diferenciar entre los dos conceptos aunque en algunos estudios se los presente como iguales, pero no es así. Los sentimientos son un estado de ánimo, y como tal estado tiene una duración variablemente prolongada.
Este se produce por causas que impresionan de forma agradable o desagradable (Damásio, 2010), y este sentimiento surge del resultado de percibir una emoción, siendo esta -según la visión expresada en la obra ética nicomaquea de Aristóteles - una emoción que puede venir acompañada de placer o de dolor (Trueba, 2009). Según la psicología la emoción es un concepto usado para explicar un proceso multidimensional que se encarga de: analizar una situación, hacer una interpretación subjetiva de la misma, expresión del proceso, preparar para la acción y produce cambios en la actividad fisiológica (Fernández-Abascal G. E, Martín M. D. \& Jiménez M. P., 2003). Las emociones básicas son: la alegría, el miedo, la tristeza, la ira, el asco y la sorpresa (Fernández-Abascal et al., 2003). Las emoción intervienen en los factores relacionados con el bienestar ver meta-análisis sobre el optimismo y la salud física: Optimism and physical Health: meta-analytic review (2009), y Optimism, Cynical Hostility and incident coronay heart disease an mortality in th women's health initiative (2009).

La Salud Mental Positiva (SMP) pretende mantener un enfoque de la Salud Mental no centrado en el déficit, sino en las partes que contribuyen a que la persona disfrute de la vida, que pueda afrontar los desafíos del día a día y además contribuir al desarrollo humano personal y comunitario. Se puede decir que Jahoda, en 1958, promulgó una visión multifactorial de la salud mental desde una perspectiva positiva. Es decir, la salud mental en su parte de normalidad clínica constituye la definición del concepto SMP (Jahoda M., 1958) . En España hay un modelo de referencia en SMP, elaborado por la Dra. Lluch-Canut (1999) que tiene como referencia el de Jahoda. Este consta de seis factores: autoconcepto, actitud prosocial, autocontrol, autonomía, resolución de problemas y autoactualización, habilidades relaciones interpersonales. Además, hay correlación entre los factores de SMP. Es decir, la falta de uno altera el desarrollo o causa déficit en otro, siendo como un circuito encadenado, entorno a la Salud Mental Positiva. Incidiendo su influencia en la SMP de la persona (Lluch- Canut, 2002). Los futuros profesionales de enfermería tienen grandes retos durante su formación, pues pasan por muchas sobrecargas de trabajo, ya que las actividades generadas durante el curso pueden causarles estrés. Asimismo, formarán parte de un grupo de riesgo en relación a bajas por trastornos afectivos, burnout, cansancio emocional, consumo de sustancias (drogas). La prevención durante la formación es una buena estrategia para proporcionar al futuro profesional herramientas para saber gestionar los factores de riesgo en beneficio de su salud. 
Hay dos vías para la promoción de atributos resilientes, una es el control de las adversidades y su impacto. La segunda vía es potenciar los atributos resilientes en los individuos vulnerables a través de la promoción en salud mental positiva. (Werner, 1992). Es decir, a través de los aspectos positivos de la salud mental. De manera que las intervenciones relacionadas con la resiliencia están focalizadas en las fortalezas, teniendo en cuenta el contexto y entorno, fomentado los recursos protectores personales y promoviendo los recursos protectores externos (Lyssenko, Rottmann \& Bengel, 2010). La salud mental positiva es un predictor de bienestar psicológico. La relación entre las emociones positivas y el cambio en la satisfacción con la vida está mediada por la mudanza en la capacidad de recuperación. Los estudios no sólo confirmaron las emociones positivas como valor predictivo de satisfacción sino también la necesidad de un aumento de estas, ya que ayudan a las personas a crear resultados deseables (Viera, López \& Barrenechea, 2006)

Observando la necesidad de trabajar las emociones en los estudiantes de enfermería, se realizó una planificación metodológica nueva, innovando tanto en la metodología como en las estrategias. Fue con la intención de adoptar una estrategia más adecuada como, después de varias búsquedas y análisis de resultados, se eligió la metodología reflexiva para trabajar las emociones.

El aprendizaje reflexivo se define de una manera constructivista; en la teoría constructivista el aprendizaje significativo, además de generar nuevos conocimientos, promueve el cambio de conducta. (Brockbank, A \& Mcgill, I., 2002). En las experiencia educativas con el uso de metodología reflexiva se ha observado buen resultado. En enfermería la utilización de las TICs como herramienta de apoyo en aprendizaje y docencia es amplia, ya que su empleo posibilita la aplicación de diferentes metodologías de aprendizaje como las PBL (Problem Based Learning), aprendizajes basados en problemas (ABP y otros...). La modalidad b-Learning (Blended Learning) en que la enseñanza es mixta (presencial y virtual) las TICS no es un simple soporte, sino que forma parte de la estrategia de aprendizaje, extendiendo este fuera del espacio físico universitario.

Con el objetivo principal de estimular la regulación emocional en los futuros profesionales de enfermería -además analizar el efecto de la nueva metodología aplicada y verificar la satisfacción de los estudiantes-, se realizó en la asignatura de enfermería de la Universidad Rovira i Virgili un proyecto piloto en la asignatura optativa de Salud Mental Positiva.

\section{METODOLOGIA}

La metodología del estudio es cualitativa fenomenológica. Muestra de 42 estudiantes del tercer curso de Grado de Enfermería de la Universidad Rovira i Virgili de Tarragona, Campus Cataluña. El rango de edad es de 21 a 53 años de edad. Siendo estos mujeres y hombres.

El instrumento de recogida de información es el espacio virtual llamado Fórum reflexivo que se realiza desde la plataforma de aprendizaje Moodle. Se analizan las actividades realizadas en el Fórum reflexivo y las observaciones realizadas durante los seminarios. El análisis del contenido fue realizado a través del método de la teoría fundamentada. El período de realización de las actividades fue de marzo de 2014 a mayo de 2014. Se realizaron siete sesiones. La identificación de la muestra es la abreviación E de estudiante y el número asignado. El fórum reflexivo:

El fórum es un espacio en el que se compartían reflexiones, conocimientos y actividades propuestas en clase. El idioma usado fue el catalán y el castellano. En él se trabajó la interacción social, reforzando las habilidades sociales y comunicativas. Además de potenciar la reflexión individual y en grupo, así como las actitudes de cooperación, comprensión y respeto entre los estudiantes. Los grupos eran seleccionados aleatoriamente a través del Moodle, cada grupo era de cuatro componentes.

Evaluación Fórum Reflexivo:

- Se evaluó la participación activa del estudiante en el fórum.

- Se valoró la realización de las actividades con muestra de conocimientos adquiridos en el aula y seminarios.

- Se evaluó el cumplimento de las pautas sugeridas.

- Se tuvo en cuenta el cumplimiento de las fechas de entrega de las actividades solicitadas, la no entrega en el plazo incidió en la nota.

Las actividades se puntuaron de 0 a 100. Era necesario el mínimo de cinco para que la actividad fuese considerada como superada. En estas actividades la evaluación fue formadora, ya que daba la posibilidad al estudiante de valorar sus aciertos y errores en el proceso de aprendizaje.

El seminario presencial:

Se elaboró una planificación de los seminarios centrada en estrategias dinámicas y que invitaba a la colaboración, análisis y reflexión. El puzzle, el rincón, trabajo de casos en grupos reducidos con tareas individuales para que cada uno tuviera un rol en la ejecución de las actividades. 


\section{RESULTADOS}

Los resultados encontrados fueron, en primer lugar el desconocimiento por parte de los estudiantes de la presencia de la emociones en la vida diaria, este desconocimiento dificulta a uno mismo reflexionar sobre sus influencias.

E. F. 32. "Es curioso como en un periodo de tiempo tan corto, (un solo día), se pueda experimentar dos tipos de sentimientos tan diferentes, los cuales evocan sensaciones muy distintas en mí."

E. F. 29. "Creo que hay que conocer los diferentes sentimientos que vamos sintiendo a lo largo de nuestra vida, interpretarlos y gestionarlos, sabiendo cuáles nos harán bien y cuáles no.”

Una vez realizada la reflexión sobre la presencia, es cuando se profundiza sobre su influencia y las posibilidades de intervenir en ellas.

E. F. 32. "Las emociones negativas nos pueden marcar el día, haciendo de él un día pésimo, pero sin darnos cuenta, también nos marcan a nosotros mismos, internamente nos sentimos tristes, deprimidos, apáticos, irritables, incluso nuestra postura es diferente, nuestra mirada es distinta, influye en el trato a los demás... en definitiva, cuando estamos de mal humor y desperdiciamos el día con la ira, la frustración, los celos y el odio, estamos desperdiciando un tiempo precioso, la vida es corta, aunque nos cueste no nos dejemos llevar por este tipo de emociones destructivas, vivámoslo sin mas...."

El trabajo de análisis e intercambio de ideas va generando nuevos conocimientos, y produciendo el aprendizaje significativo, que es cuando lo que aprendes traslada a la vida real.

E. F 29. "Pienso, al igual que..., que tanto el concepto de Resiliencia como la gestión de los sentimientos van de la mano la mayoría del tiempo. Uno no puede ser resiliente si no tiene una base mínima de respuesta adaptativa con un sustento de sentimientos positivos que le impulsen al cambio y a sobreponerse a la situación o el hecho que le ha llevado a donde está."

E. F. 21. Bon dia ...., després de llegir la reflexió que fas sobre l'afectació dels sentiments i emocions a la teva vida observo que en cada situació viscuda durant el transcurs del dia experimentes diferents sentiments $i$ emocions, passant des de la por i hostilitat matinal, a l'hora de passejar el gos, fins a la sensació de benestar que et genera quan et trobes amb la teva parella i el sentiment de felicitat."
E. F. 29. Darte cuenta de cómo influyen tus reacciones sobre los demás y a viceversa ayuda a entender por qué se plantean algún tipo de situaciones. Si reflexionásemos más sobre esto, seguro que en general resolveríamos muchos más conflictos"

La mejora de autoconcepto al exponer sus dificultades y recibir el apoyo y comprensión por parte del grupo.

E. F. 26. “..., entiendo tu situación, tal vez desde otra perspectiva, pero entiendo el sentimiento que tienes en relación a tu trabajo. Creo que... tienes razón, en lo que dice. En la actividad dices que tu forma de luchar contra los sentimientos negativos que te crea tu trabajo es equilibrarlos con los buenos sentimientos que tienes durante el día con tu novio y familia"

E. F. 12. "Creo que tal vez podría intentar, como dice Ángel, mirar la situación que te crea malos sentimientos de otra manera. Por ejemplo, cuando yo trabajaba de noches, me marcaba metas, como por ejemplo, hacer cualquier cosa mejor, que quede mas bonita o en menos tiempo. Puede parecer una tontería, pero yo los veo como pequeños objetivos que te obligan a mejorar."

E. F. 28. "Me ha gustado mucho leer la reflexión de... está repleta de actitudes dignas de imitar, gracias por tu aportación...tan personal. A mí también me sucede lo mismo que a vosotras... en cuanto estamos saturados por aspectos de la vida no universitaria y la vida universitaria, siento como me saturo y el tiempo se escurre entre mis dedos sin poder avanzar."

La satisfacción de los estudiantes por haber participado en un espacio virtual donde no solo se reflexionaba acerca de lo aprendido en el seminario sino que además se aportaba y compartían sus experiencias y emociones. También despertar el deseo de continuar se auto-actualizando.

E.F. 21."Esta asignatura me doy cuenta, cada vez más, lo importante que es que la gente trabaje e invierta energía en cuidar su salud mental. En el futuro el trabajo como enfermeros estaría bien aprovechar esa oportunidad para motivar a las personas a autorrealizarse, que aun teniendo una enfermedad o pasar por un proceso patológico temporal, se sientan a gusto con ellos mismos y puedan usar esos procesos como una oportunidad de trabajar la resiliencia y conocerse mejor."

E.F. 03. "Us agraeixo de tot cor la vostra sinceritat al comentar els meus escrits i espero que hagueu tingut una bona experiència, tal i com ha sigut la meva." 


\section{DISCUSIÓN}

El proceso de autorreflexión que posibilitó la estrategia b-learning/Fórum reflexivo promovió las expresiones de las emociones, haciendo que el estudiante tomara consciencia de su presencia, importancia e influencia. Las expresiones de emociones en contextos inapropiados están asociados a problemas con la adaptación social (Cole, P. M., Miche, M. K. \& Teti, L. O., 1994).

Los estudiantes pasan por un periodo formativo que les genera mucho estrés. El hecho de haber tenido un espacio para compartir estas preocupaciones y elaborar otro punto de vista sobre el tema ayuda a dar una visión más optimista de la experiencia. El optimista tiene la capacidad de valorar las estrategias de afrontamiento dirigido al problema y en caso de que no haya posibilidad de solucionarlo recurre a las estrategias centrada en las emociones (el humor, reinterpretación positiva de la vivencia, buscar apoyos para amortiguar los efectos negativos (Ortiz, Ramos \& Villarroel, 2003). Además, las emociones positivas sirven para moderar el efecto del estrés y mediar en la recuperación, nunca para eliminar el estrés ya que es un elemento presente en la vida humana (Tindle et al., 2009). La regulación emocional aumenta las habilidades personales y favorece la vida social (Brackett, M., Rivers, Shiffman, S., Lerner N. \& Solovey P, 2006). En este proyecto los resultados han sido muy parecidos a los estudios que trabajan las competencias emocionales en relación a promoción y prevención, metodologías como el Aprendizaje Social y emocional (SE). Se trata de un proceso que integra cinco competencias básicas: autoconciencia, la autorregulación, la automotivación, la empatia y la relaciones interpersonales (Durlak A. J., Weissberg P. R., Shellinger B.K., Taulor R.D. \& Schellinger K.B, 2011).

\section{CONCLUSIÓN}

La conclusión del estudio fue que la metodología resultó efectiva, el empleo de la metodología b-Learning posibilitó la expresión de emociones, tanto por escrito como presencial (Seminarios). Además de promover los pasos para la competencia emocional (conciencia de propio estado emocional, utilizar vocabulario emocional, empatía, capacidad de autoeficacia emocional...). La participación en un espacio virtual fomentó las relaciones interpersonales, ya que no sólo se conocieron mejor, sino que también compartieron sus dificultades y transferencia de apoyo y comprensión. Además de promover el autoconcepto, ayudando a ver una autoimagen más positiva de uno mismo, a través de las valoraciones positivas de los compañeros.
El fórum promovió el autoconocimiento, y como resultado, el descubrimiento de puntos fuertes y otros que necesitan ser trabajados en relación a las emociones. Este análisis es importante para los cambios de conducta saludable. Aunque la muestra es pequeña para generalizar los resultados, sirve como línea para futuros proyectos de innovación o promoción y prevención en salud mental.

\section{IMPLICACIONES PARA LA PRATICA CLINICA}

El método b-Learning es una estrategia muy fácil, práctica y de fácil valoración. En el trabajo de promoción y prevención en salud mental, los recursos virtuales pueden ejercer un rol importante ya que, aunque las sesiones presenciales tienen relevancia, el espacio virtual se traslada fuera del tiempo y el espacio físico reglado. Facilitando así el acceso y motivando la participación en los programas de prevención y promoción.

\section{REFERENCIAS BIBLIOGRÁFICAS}

Brackett, M., Rivers, Shiffman, S., Lerner N., \& Solovey, P. (2006). Relating emocional abilities to social funtioning: A comparison of self-reporte and performance measures of emotion intelligence. Journal of Personality and Social Psychology, 91(4), 780-795.

Brockbank A., \& Mcgill I. (2002). Aprendizaje reflexivo en la educación superior. Madrid, España: Ed. Morata.

Cole, P. M., Miche, M. K., \& Teti, L. O. (1994). The developament of emotion regulation and dysregulation: clinical perpective. Monografs of the Society Research in Child Developament, 59(2-3), 73-100.

Damásio, A. (2010). Self Comes to Mind: constructing the Consciens Brain. New York, United States of America: Vintage Books.

Diener, E. (2000). Subjetive Well-being: The Science of Happiness and a proposal for a national index. Journal American Psychologist, 55(1), 34-43.

Dimitry M. D., Stewart R., Ritchie K., \& Cahudieu I. (2010). Resilience and Mental Health. Clinical Psychology Review, 30(5), 479-495. doi: 10.1016/j. cpr.2010.03.003 
Durlak A. J., Weissberg P. R., Shellinger B. K., Taulor R. D., \& Schellinger K. B. (2011). The impact of ennhancing students social and emotional learning: A metaanalysis of school-based universal interventions. Child Development, 82(1), 405-432. doi: 10.1111/j.14678624.2010.01564.x

Fernández-Abascal G. E, Martín M. D., \& Jimenez M. P. (2003). Emoción positiva y motivación. Madrid, España: UNED.

Jahoda M. (1958). Current Concepts of Positive Mental Health. Acedido em 22 de setembro, 2015, em https://archive.org/details/currentconceptso00jaho

Lyssenko, L., Rottmann, N., \& Bengel, J. (2010). Resilienzforschung. Acedido em 5 de setembro, 2015, em http:// www.fruehehilfen.de/fileadmin/user_upload/fruehehilfen.de/pdf/Bundesgesundheitsblatt_Artikel_Bengel.pdf

Lluch-Canut, M. T. (2002). Evaluación empírica de un modelo conceptual de Salud Mental Positiva. Salud Mental, 25(4), 42-55.

Lyssenko, L., Rottmann, N., \& Bengel, J. (2010). Research on psychological resilience. Relevance for prevention and health promotion. Bundesgesundheitsblatt Gesundheitsforschung Gesundheitsschutz, 53(10), 1067-72.

Organización Mundial de la Salud (2014). Definición de salud mental. Acedido a 2 de setembro de 2015, em http:// www.who.int/topics/mental_health/es/
Ortiz, J., Ramos, N., \& Villarroel, P. V. (2003). El optimismo y la salud: Estado actual e implicaciones para la psicología clínica y de la salud. Suma Psicológica, 10(1), 119-134.

Rasmussen H. N., Sheier M. F., \& Greenhouse J. B. (2009). Optimism and physical health: Meta-analytic review. Annuary Behavior Medicine, 37(3), 239-256.

Ryff, C. D. (1989). Happiness is everything, or is it? Explorations on the meaning of psychological well-being. Journal of happiness studies. Acedido a 10 de setembro de 2015, em http://sccn612final.wikispaces.com/file/ view/Hedonia,+Eudaimonia-+An+Introduction.pdf

Tindle H. A., Chang Y. F., Kuller L. H., Manson J. E., Robinson J. G., Rosal M. C., \& Matthews K. A. (2009). Optimism, Cynical Hostility and incident coronay heart disease an mortality in the women's health initiative, 120(8), 656-662. doi: 10.1161/CIRCULATIONAHA.108.827642

Trueba A. C. (2009). La teoría Aristotélica de las emociones. Signos Filosóficos, 11(22), 147-170. Acedido a 20 de setembro de 2015, em http://www.redalyc.org/articulo.oa?id=34316032007

Viera, A. O., López, S. S., \& Barrenechea, A. V. (2006). The psychological well-being a positive indicator of mental health. Revista Cubana, 7(1-2), 34-39.

Werner, E. E. (1992). The Children of Kauai: Resiliency and recovery in adolescence and adulthood. Journal of Adolescence Health, 13(4), 262-268. doi: 10.1016/1054139X(92)90157-7 